\title{
Energy Technology Environment Model with Smart Grid and Robust Nodal Electricity Prices*
}

\author{
Frédéric Babonneau $^{\dagger} \quad$ Alain Haurie $^{\ddagger}$
}

May 25, 2016

\begin{abstract}
In this paper one introduces the modeling of power flow constraints for transmission grid in the multi-sectoral multi-energy long-term investment planning tool ETEM-SG. This extension allows a better representation of demand response for flexible loads triggered by real time marginal cost pricing. Since the loads and the generation units are geographically distributed these prices should be represented by nodal marginal costs. Now ETEM-SG contains a linearized DC power flow model that represent the transmission grid with the main constraints on the power flowing through the different arcs of the electricity transmission network. Robust optimization is used to take into account the uncertainty on the capacity limits resulting from inter-regional transit. A numerical illustration is carried out for a data set corresponding grossly to the Leman Arc region.
\end{abstract}

\section{Introduction}

The transition to sustainable energy system, in Europe as in the rest of OECD countries involves an increase of distributed power generation from variable renewable sources such as wind turbines and solar panels, the development of electric mobility, the linking of power and heat or cooling generation and the active use of demand response. ETEM-SG is a model developed recently [3, 16] to assess the future role of renewable and smart-grid technologies in the energy transition, at a regional level.

In this paper one presents an extension of the multi-sectoral multi-energy long-term investment planning tool ETEM-SG permitting the consideration of power flow constraints in the regional transmission grid. This extension is needed because demand response is modeled as an optimal response of flexible loads to marginal cost pricing. Since the loads and the generation units are geographically distributed these prices should be represented by nodal marginal

\footnotetext{
*This research is supported by the Qatar National Research Fund under Grant Agreement no 6-1035-5-126.

${ }^{\dagger}$ ORDECSYS and LEURE Laboratory, Swiss Federal Institute of Technology at Lausanne (EPFL)

${ }^{\ddagger}$ ORDECSYS, Place de l'Etrier 4, CH-1224 Chêne-Bougeries, Switzerland.
} 
costs associated with a representation of the transmission grid. A linear DC-equivalent power flow model is now included in ETEM-SG, providing a representation of the transmission grid with the main constraints on the power flowing through the different arcs of the electricity transmission network. The scenarios obtained through running ETEM-SG will thus propose, for each time slice an optimal dispatch of production units of the regional energy system with demand response activities triggered by marginal cost pricing, and at a larger time scale, the optimal location and timing of new capacities introduction for power generation (in particular the technologies based on renewable sources), the development of distributed storage (e.g. through electric cars and PHEVs) and the investment in network reinforcement.

Because the power flows circulating in a regional transmission grid depend on what happens on the transmission grid for a much larger perimeter, a robust optimization (RO) technique [8, 6] is introduced to take into account the resulting uncertainty on the capacity limits for the different arcs of the regional transmission grid. RO is an alternative to classical approaches (eg, Stochastic Programming, Chance Constraint Programming) that aims at overcoming numerical issues induced by calculus of probability and by the well-known curse of dimensionality. The main idea of RO is to start with a non-probabilistic formulation of uncertainty, namely the uncertainty set, and look for solutions that remain satisfactory for all possible realizations in the uncertainty set. Solutions having this property are named robust. As no probability model is assigned to the uncertainty, computing robust solution becomes a numerically tractable operation. The paradigm of robust linear optimization goes back to [20] and it has been revived in the nineties by El-Gahoui and Lebret [12] and by Ben-Tal and Nemirovski [9]. Recently it has been applied to long-term energy models to cope with different sources of uncertainties. In [6], the authors combined RO with Stochastic Programming in a power supply model under pollution constraints with uncertainties on demands and pollutants diffusion coefficients. Energy security of EU is analysed in [4] using the long-term TIAM-WORLD model in which energy supply routes are subject to random events. In [1], RO is also applied to deal with uncertainty related to the impacts of climate change on the evolution of regional energy systems.

The paper is organized as follows. In Section 2, one gives a brief presentation of the multi-sectoral multi-energy long-term investment planning tool ETEM-SG. In Section 3, one describes the linearized DC power flow model to be introduced in ETEM- SG and one addresses the implementation issues. Section 4 is devoted to the robust optimization approach to deal with uncertain power flow transits. In Section 5 , a numerical illustration is provided and Section 6 we concludes.

\section{ETEM-SG in short}

The reader is referred to [2] for a complete description of the ETEM-SG model. ETEM-SG is a linear programming model, related to the MARKAL/TIMES family of models [10, 11, 13], which represents the optimal capacity expansion in production technology and the flow of resources in the whole energy system. ETEM-SG is a multi-sectoral, multi-energy, technology rich model (See Figure 1). 


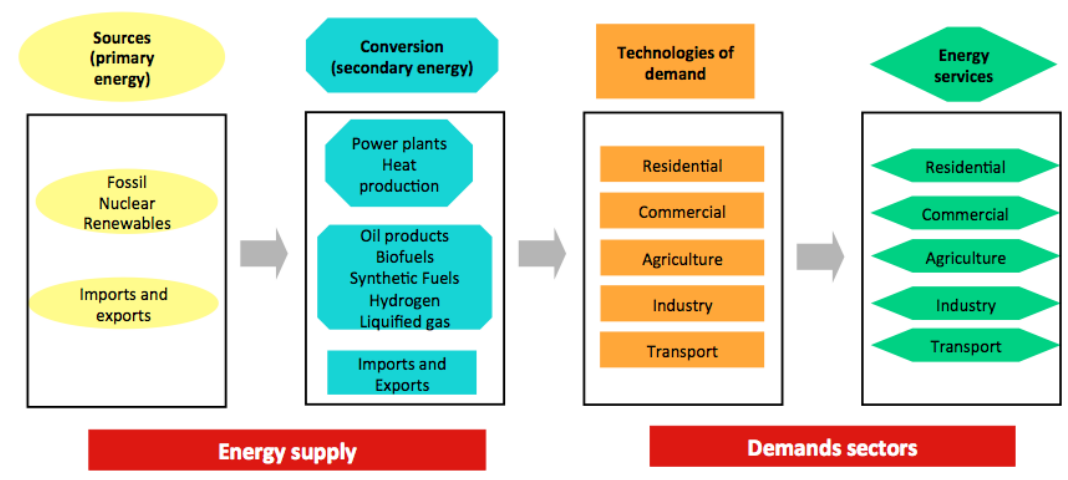

Figure 1: Reference Energy System

In its standard version, the model is driven by exogenously defined useful energy demands, that is the demand for energy services, and imported energy prices. All technologies are defined as resource transformers and are characterized by technical coefficients describing input and output, efficiency, capacity bounds, date of availability (for new technologies), life duration, et. Economic parameters define investment, operation and maintenance costs for each technology. The planning horizon is generally long enough to offer a possibility for the energy system to have a complete investment technology mix turnover.

Typically ETEM simulates the development of an efficient regional energy system with a planning horizon of 30 to 50 years usually divided in periods $t \in T$ of 1 to 5 years. In each period one considers a few typical days (e.g., 6 days corresponding to the three seasons Winter, Summer, Spring-Fall - and two week day types - working weekday, weekend-Holiday - ). Each of these days is subdivided into groups of hours, to obtain finally a set of timeslices $S$ that will be used to represent load curves, distribution of demand and resource availability in different seasons and at different time of the day. This time structure is particularly important to represent correctly demands dynamics and the way one can exploit their flexibility. This mechanism is known as demand-response. The definition of timeslices in ETEM-SG should allow a representation through state equations of the dynamics of the energy services required during a day, like e.g. maintaining a comfort zone in residential heat and recharging EVs and PHEVs. i

\section{Modeling optimal power flows in ETEM-SG}

This section deals with the introduction of a linearized DC power flow model in ETEM-SG and addresses some implementation issues. For more details, the reader is referred to [7] where the modeling of transmission grid constraints and optimal power flows is well described. 


\subsection{Linearized load flow model}

Consider a transmission network with $N$ nodes (or buses) linked by $L$ lines, described by the following variables and parameters:

$y_{j}:$ net power injection at node $j=1, \ldots, N$; $\mathbf{y}$ is the $N$ vector with elements $y_{j}$.

$z_{\ell}$ : flow along line $\ell=1, \ldots, L ; \mathbf{z}$ is the $L$ vector with elements $z_{\ell}$.

$\bar{A}:$ network incidence matrix $L \times N$, with $a_{\ell, j}=1$ if line $\ell$ originates from $j, a_{\ell, j}=-1$ if line $\ell$ terminates on $j, a_{\ell, j}=0$ otherwise. Note that the sum of the columns of $A$ is always equal to the the null column.

A: an $L \times(N-1)$ matrix obtained by removing a column corresponding to the swing bus in the matrix $\bar{A}$.

S: an $L \times L$ diagonal matrix, $\mathbf{S}=\operatorname{diag}\left(S_{1}, \ldots, S_{L}\right)$, where $S_{l}$ is the susceptances 11 matrix of line $l$.

The linearized Power Flow equation can be written as

$$
\mathbf{z}=\mathbf{S} A \theta
$$

where $\theta$ is the $N$-vector of angles at the different nodes (buses). Since $\mathbf{y}_{-}=A^{T} \mathbf{z}$, and by introducing $A^{T} S A$, one gets:

$$
\mathbf{z}=\mathbf{S} A\left(A^{T} \mathbf{S} A\right)^{-1} \mathbf{y}_{-},
$$

which can be rewritten

$$
\mathbf{z}=\Psi \mathbf{y}_{-},
$$

where $\Psi$ is now called the injection shift factor matrix.

\subsection{The optimal dispatch problem and its dual solution}

Assume that an accurate description of the transmission grid is obtained by using a linearized AC load flow model and neglecting losses on the lines. The nodal prices can be obtained from the dual solution of an optimal dispatch problem under constraints of capacity of the generators and transmission network, as shown by Ruiz et al. [17, 18, 19] or Stiel [21].

The distribution of power in the different lines of the transmission network is given by Eq. (2) which we rewrite as follows

$$
P_{f}=\Psi\left(P_{G}-P_{L}\right),
$$

\footnotetext{
${ }^{1}$ In electrical engineering, susceptance $(B)$ is the imaginary part of admittance. The inverse of admittance is impedance and the real part of admittance is conductance. In SI units, susceptance is measured in siemens.
} 
where $P_{f}=\mathbf{z}$ is the vector of power flows on each line of the network and $P_{G}-P_{L}=\mathbf{y}$ is the vector of net power injection (generation power $P_{G}$ minus load $P_{L}$ ) at each bus (node) of the network. The transmission sensitivity matrix $\Psi=\mathbf{S} A\left(A^{T} \mathbf{S} A\right)^{-1}$, also known as the injection shift factor matrix, gives the variations in flows due to changes in the nodal injections. The shift factor matrix is a function of the characteristics of the transmission elements and of the state of the transmission switches. For a given point in time, the system operator dispatches the committed units so as to minimize the total costs of operations. Assume that the generation costs are piecewise linear, and denote the vector of nodal generation annualized costs ${ }^{2}$ by $c_{G}$.

The economic dispatch is formulated as the following linear program:

$$
\min _{\left\{P_{G}\right\}} c_{G}^{T} \cdot P_{G}
$$

under the following set of constraints (with the associated dual variables indicated in the RHS):

$$
\begin{aligned}
\mathbf{1}_{N}^{T}\left(P_{G}-P_{L}\right) & =0 \leftrightarrow \lambda, \\
P_{f \min } \leq \Psi\left(P_{G}-P_{L}\right)_{-} & \leq P_{f \max } \leftrightarrow \mu_{\min }, \mu_{\max }, \\
P_{G \min } \leq P_{G} & \leq P_{G \max } \leftrightarrow \gamma_{\min }, \gamma_{\max },
\end{aligned}
$$

where $\mathbf{1}_{N}$ stands for an $N$ vector whose components are all equal to 1 . The constraint (6) ensures the total load-generation balance, (7) enforces the flow limits on transmission elements and flowgates, where lower limits usually represent the limit in the opposite flow direction, and (8) models the lower and upper generation limits. In [19], it is shown that the nodal marginal prices is then given by

$$
\pi=-\left(\lambda \mathbf{1}+\Psi^{T}\left(\mu_{\max }-\mu_{\min }\right)\right) .
$$

One must now introduce the optimal dispatch model in a multi-energy long term LP model like ETEM-SG [3]. The implicit nodal prices given by expressions similar to (9) in this larger model will then serve to guide demand response, e.g. in charging of EVs or PHEVs and the use of these technologiesfor distributed storage.

\subsection{Introduction of a transmission grid sub-model in ETEM-SG}

The optimal dispatch equations (with a proper representation of the power transmission grid) are introduced in the ETEM-SG equations at the finest level of time scale and geographical information.

Time scales representation. The dispatch problem is to be solved explicitly in ETEM-SG for every time slices $s \in S$ and all periods $t \in T$ under regular and peak load conditions. Power flows on the transmission grid are thus computed for all time slices.

Geographical decomposition. Because the transmission grid defines some important constraints in the dispatch problem, it is necessary to decompose the regional energy system represented in ETEM-SG in subregions $n \in N$, each one corresponding to a node of the transmission grid. Useful demands are defined for each subregion separately and ETEM-SG will

\footnotetext{
${ }^{2}$ expressed in $\$ / \mathrm{MWh}$ or $\mathrm{CHF} / \mathrm{MWh}$.
} 
thus determine a complete energy sub-system at each node of the grid, describing in particular, electricity production units and technologies generating an electricity demand. Then electricity generation (injections) and loads are computed at each node for each time slices and used to determine the resulting power flow.

Power flow equations in ETEM-SG. The new equations introduced in ETEM-SG and the link with existing variables and constraints are described here: Let $N$ be the set of subregions represented in ETEM-SG and $\bar{L}$ the number of pairs of subregions that are connected and that may directly exchange electricity. Note that $\bar{L} \leq L, L$ being the number of transmission lines, as two connected subregions can be linked by multiple line transmissions. In the standard ETEM-SG formulation, there is a variable, denoted Exchange $\left[t, s, n_{1}, n_{2}\right]$, that represents the electricity energy exchanged between subregions $n_{1} \in N$ and $n_{2} \in N$ in period $t$ and timeslice $s$. A positive number means electricity goes from $n_{1}$ to $n_{2}$ while a negative one means the opposite.

The following new equation constraints links power flow variables with the electricity exchanges:

$$
\text { Exchange }\left[t, s, n_{1}, n_{2}\right]=\alpha^{s} \sum_{l \in L_{n 1, n 2}} z_{l}^{t, s}, \quad \forall t \in T, \forall s \in S, \forall n_{1} \in N, \forall n_{2} \in N .
$$

where $L_{n_{1}, n_{2}} \subset L$ is the subset of arcs between $n_{1}$ and $n_{2}, z_{l}^{t, s}$ is the power flowing from $n_{1}$ to $n_{2}$ (or from $n_{2}$ to $n_{1}$ if negative) at period $t$ and timeslice $s$, and $\alpha^{s}$ is a coefficient to convert energy to power.

Each power flow is constrained by line capacities $c$

$$
-c_{l}^{t} \leq z_{l}^{t, s} \leq c_{l}^{t}, \quad \forall t \in T, \forall s \in S, \forall l \in L .
$$

Finally power flows are defined from equations (1):

$$
z_{l}^{t, s}=\left(\theta_{n_{1}}^{t, s}-\theta_{n_{2}}^{t, s}\right) s_{l}, \quad \forall t \in T, \forall s \in S, \forall l \in L
$$

where $\theta$ are variables representing bus angles at transmission nodes, and $s_{l}$ is the susceptance factor of line $l$.

\section{Robust optimization to deal with uncertain power flow transits}

The power flowing through a regional transmission grid depends on regional activity but also on what happens on the transmission grid on a much larger perimeter du to power flow transits. When dealing with long-term analysis, these activities are uncertain but have an impact on regional network congestion. An approach for simulating these power flow transits would consist in using flow estimates from a model representing the aforementioned larger perimeter. Without a proper access to this information, it is proposed here the use a robust optimization 
approach to take into account the resulting uncertainty on the capacity limits for the different arcs of the regional transmission grid.

In so doing, the randomness of the situation is formulated broadly. In other words, one does not model uncertain capacity on each transmission line separately but, instead, the entire set of lines is considered simultaneously when assessing the risk. This is justified by the fact that the modeler is not interested in knowing exactly what happens on each individual line but, rather, in defining power flows that may satisfy regional loads and injections at nodes at all time slices and for all possible conditions of lines saturation.

One therefore creates aggregate capacity constraints by summing at each timeslice $s \in S$ and period $t \in T$ the $|L|$ constraints $(10)$ to obtain :

$$
-\sum_{l \in L} \beta_{l}^{t} c_{l}^{t} \leq \sum_{l \in L} z_{l}^{t, s} \leq \sum_{l \in L} \beta_{l}^{t} c_{l}^{t}, \quad \forall t \in T, \forall s \in S,
$$

where $\beta_{l}^{t}$ are random factor with values in $[0,1]$. For the sake of simpler notations, the time indices $s$ and $t$ are omitted in the following equaions.

Remark 1. The present application is, at each timeslice, a dynamic two-stage problem under uncertainty. In such problems, successive decisions can be made on the basis of the information revealed so far. In our case, it means that power flows should be adjusted automatically to power transit. In other words, the second step decisions (power flows) are contingent on the outcome of the uncertain parameters. There exist techniques to model such a dynamic feature (eg, Affine decision rules) but, unfortunately, these techniques introduce an order of complexity that leads large-scale models such as ETEM-SG to intractable formulation. This paper does not tackle the second-level decision making and refers the reader to [6] 5] for a more detailed discussion on uncertainty in dynamic models.

\subsection{Uncertainty model}

Define the random coefficients $\beta_{l}$ as follows

$$
\beta_{l}=\bar{\beta}_{l}-\hat{\beta}_{l} \xi_{l}
$$

where $\bar{\beta}_{l}$ represents the nominal congestion rate of transmission line $l$ resulting from power

transits, $\hat{\beta}_{l}$ the congestion variability and $\xi$ is a set of independent random variables with support $[-1,1]$. Using this definition, the capacity of line $l$ available locally takes values in $\left[c_{l}\left(\bar{\beta}_{l}-\hat{\beta}_{l}\right) ; c_{l}\left(\bar{\beta}_{l}+\hat{\beta}_{l}\right)\right]$. Eq. 111 becomes:

$$
-\sum_{l \in L}\left(\bar{\beta}_{l}-\hat{\beta}_{l} \xi_{l}\right) c_{l} \leq \sum_{l \in L} z_{l} \leq \sum_{l \in L}\left(\bar{\beta}_{l}-\hat{\beta}_{l} \xi_{l}\right) c_{l}
$$

which can be written differently as:

$$
\begin{aligned}
& \sum_{l \in L}\left(\bar{\beta}_{l} c_{l}+z_{l}\right)-\sum_{l \in L} \hat{\beta}_{l} \xi_{l} c_{l} \geq 0 \\
& \sum_{l \in L}\left(\bar{\beta}_{l} c_{l}-z_{l}\right)-\sum_{l \in L} \hat{\beta}_{l} \xi_{l} c_{l} \geq 0,
\end{aligned}
$$


where the first summation of two constraints is linear deterministic expressions whereas the second summations represent random terms.

\subsection{Robust optimization for ETEM-SG}

One applies Robust Optimization method [8] to $113 \mathrm{a}$ and $(13 \mathrm{~b}$. Although constraints are immunized separately in the Robust Optimization paradigm, it has been showed in [6, 5] that two-sided inequality constraints such as $(13 \mathrm{a})$ and $(13 \mathrm{~b})$ can be treated simultaneously.

One considers an uncertainty set defined as follows :

$$
\Xi=\left\{\xi \mid-1 \leq \xi_{l} \leq 1 \text { and } \sum_{l \in L} \xi_{l} \leq k\right\}
$$

Using robust optimization techniques, the worst case situation for network saturation is given by the robust equivalent of the robust constraints (13a) and (13b)

$$
\begin{aligned}
& \sum_{l \in L}\left(\bar{\beta}_{l} c_{l}+z_{l}\right)-k\left\|\hat{\beta}_{l} c_{l}-w\right\|_{\infty}-\|w\|_{1} \geq 0 \\
& \sum_{l \in L}\left(\bar{\beta}_{l} c_{l}-z_{l}\right)-k\left\|\hat{\beta}_{l} c_{l}-w\right\|_{\infty}-\|w\|_{1} \geq 0
\end{aligned}
$$

which is equivalent to the system of linear inequalities

$$
\begin{aligned}
\sum_{l \in L}\left(\bar{\beta}_{l} c_{l}+z_{l}\right)-\sum_{l \in L} u_{l}-k v & \geq 0, \\
\sum_{l \in L}\left(\bar{\beta}_{l} c_{l}-z_{l}\right)-\sum_{l \in L} u_{l}-k v & \geq 0, \\
u_{l}+v & \geq \hat{\beta}_{l} c_{l}, \quad \forall l \in L,
\end{aligned}
$$

with additional variables $u$ and $v$.

From [8], one can derive a satisfaction probability for capacity constraints for any realizations of $\xi \in \Xi$ that depends on the radius $k$ of the uncertainty set. This result is given in Proposition 1. The factor $k$ plays a crucial role as the larger its value, the greater the number of realizations $\xi$ that are considered.

Proposition 1. Let $\xi_{i}, i=1, \ldots, n$ be independent random variables with values in interval $[-1,1]$ and with average zero: $E\left(\xi_{i}\right)=0$. Then, for all $k \geq 0$

$$
\operatorname{Prob}\left\{\xi \mid \sum_{l \in L} \hat{\beta}_{l} \xi_{l} c_{l}>k\left\|\hat{\beta}_{l} c_{l}-w\right\|_{\infty}\right\} \leq \exp \left(-\frac{k^{2}}{2.5|L|}\right) .
$$

In the numerical experiment presented in Section 5, one considers a transmission network with 12 lines (ie, $|L|=12$ ) and one sets $k=10$. This leads to a $96 \%$ constraint satisfaction probability. 


\section{Numerical illustration}

To illustrate the model a case study has been developed, which corresponds broadly to the regional energy system of the "Léman Arc" area in Switzerland (Cantons of Vaud and of Geneva). Note that the objective of this numerical simulations is to illustrate the impact of introducing a representation of power flow constraints and robustness in a regional energy model, and not to provide a precise representation of the energy policy choices in this region.

The energy model is adapted from an ETEM model that had been developed in previous project 5 in which three subregions were represented. This spatial decomposition corresponds, globally, to the three power distribution companies operating in the region (i.e., $S I G$ for Geneva, SIL for Lausanne and Romande Energie (ROM) for the rest of the region) but it does not match any grid transmission aspect. One explains below how the regional energy system has been reorganized into 9 subsystems connected through power transmission lines.

\subsection{Data}

Energy system of Lac Léman area. In 2010, the total annual energy consumption of the "Léman Arc" region was $114.3 \mathrm{PJ}$ and, overall, $\mathrm{CO}_{2}$ emissions amounted to $5.48 \mathrm{Mt}$. The region is a net importer of electricity, around 5.5 TWh out of a total electricity consumption of 7.1 TWh in Year 2010 4

Transmission grid. Figure 2 shows the Swiss transmission grid as reported in [22]. The network used in the ETEM-SG model will be a subgraph of this network, i.e., the one corresponding to the bottom-left corner. From the description of the swiss transmission grid one can extract the sub-grid involved in the "Léman Arc" area. It is schematically represented in Figures of resuls 7 and 8 with 9 nodes each one connected to a local energy subsystem and 12 transmission lines. Among the 9 nodes, three (Verboix, Romanel and Triphon) are connected to the Swiss and European transmission grid for electricity import/export and transit. Line capacities and reactances are set to values used in [22].

Useful demands. Table 1 gives the regional useful demands considered in the case study and Figure 3 displays their assumed evolution up to 2050. For the present exercise, demands are distributed geographically among the 9 subsystems connected to the transmission grid. The allocation of demand to nodes is obtained by first satisfying the observed demands for the three power distribution companies in the three main areas (Geneva, Lausanne and the rest of the region) and then by distributing uniformly the demand to the buses located in the considered areas ( 2 buses for Geneva, 2 buses for Lausanne and 5 buses for the rest of the region).

Finally demands are distributed on a yearly basis, among the 12 timeslices defined, for three seasons (Winter, Summer, Intermediate), and four parts of Day (Night, morning peak P1,

\footnotetext{
${ }^{3}$ The reader is referred to the RITES [15] and TOU [16] projects, which were supported by the Swiss Federal Office of Energy.

${ }^{4}$ For more details on the global energy system, the reader is referred to [16, 15].
} 


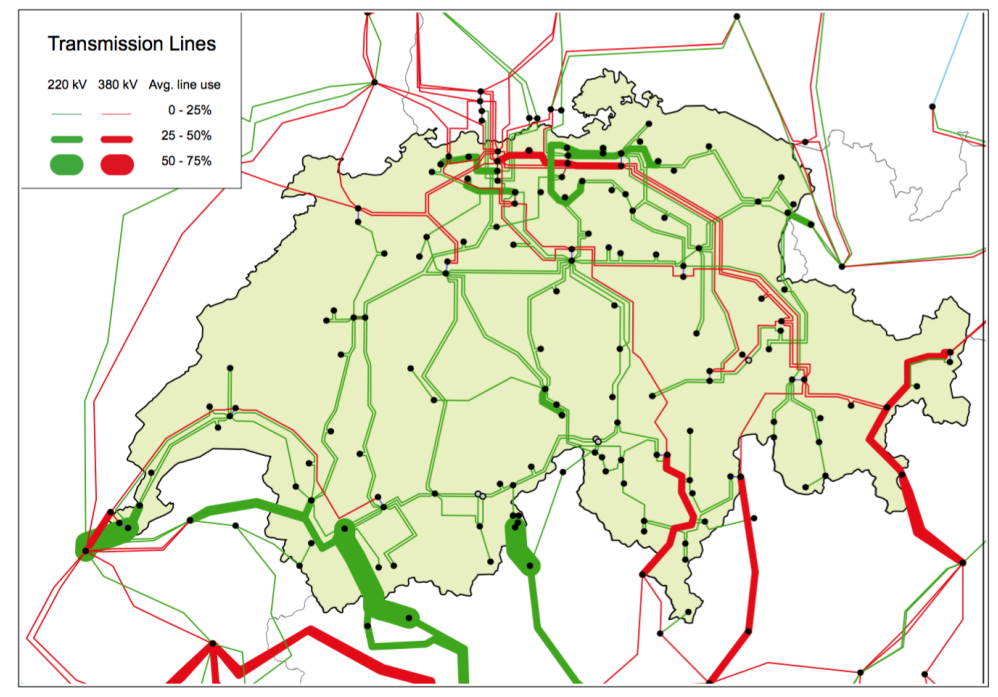

Figure 2: Swiss transmission grid in 2015 (Figure from [22])

Mid-Day and evening peak P2), as illustrated in Figure 4.

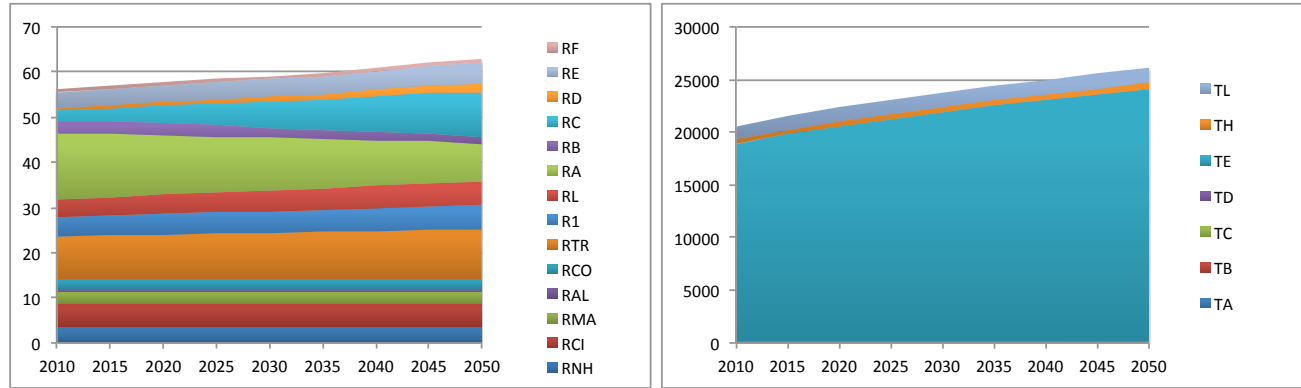

Figure 3: Evolution of useful demands in PJ (left) and in tkmv/d (right).

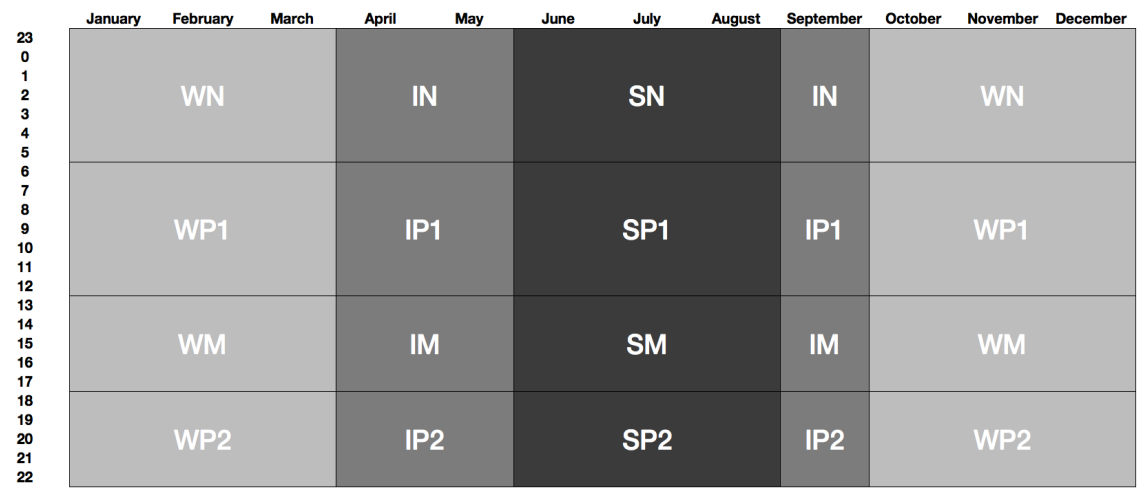

Figure 4: Definition of timeslices 


\begin{tabular}{llll}
\hline Sector & Label & Code & Unit \\
\hline \multirow{2}{*}{ Residential } & Heat Existing Buildings 2-9 appts & RA & PJ \\
& Heat Existing Houses & RB & PJ \\
& Heat New Buildings 2-9 appts & RC & PJ \\
& Heat New Houses & RD & PJ \\
& Appliances & R1 & PJ \\
& Lighting & RL & PJ \\
\hline \multirow{2}{*}{ Transport } & Public Transports: Bus & TA & tkmv/d \\
& Public Transports: Tramway & TB & tkmv/d \\
& Public Transports: Train & TC & tkmv/d \\
& Public Transports Misc. & TD & tkmv/d \\
& Automobile & TE & tkmv/d \\
& Truck & TH & tkmv/d \\
& Delivery vehicles & TL & tkmv/d \\
\hline \multirow{2}{*}{ Industry } & Food, textile, wood, paper, edition & RNH & PJ \\
& Chemistry, rubber, glass, metal & RCI & PJ \\
& Machine manufacturing, equipments & RMA & PJ \\
& Construction & RCO & PJ \\
& Tertiary & RTR & PJ \\
& Other & RAL & PJ
\end{tabular}

Table 1: Useful demands classification (PJ means PetaJoule and tkmv/d means thousand $\mathrm{km}$ vehicle per day).

\subsection{Scenario definition}

The swiss energy strategy scenario. The Swiss Federal Office of Energy (SFOE) has proposed a scenario for energy transition, called Neue Energiepolitik (NEP). It describes the Swiss Energy Strategy at horizon 2050 [14]. We use similar boundary assumptions to those in NEP for the three scenarios developed with ETEM-SG for "Léman Arc" area. These scenarios will illustrate the importance of taking into consideration power flow constraints at a regional scale. In particular, in the NEP scenario, the emissions of greenhouse gases are caped at a level of 1.5 tons of $\mathrm{CO}_{2}$-eq per person in 2050. Since the population is expected to attain $1.37 \mathrm{M}$ people in the Arc Lémanique region by 2050 ('mittleres' Szenario A-00-2010), we impose as a constraint that the total 2050 emissions should not exceed $2.1 \mathrm{Mt} \mathrm{CO}_{2}$-eq in the region.

Simulating network congestion. In order to evaluate the impact of power flow constraints and in particular of network congestion on simulation results, three different network settings are compared.

- In the first scenario, it is assumed that the full existing line capacities (i.e., $490 \mathrm{MW}$ ) is available for transmission in the region. Without considering inter-regional power transit, the proposed network is oversized and thus congestion does not occur.

- In the second scenario, one assumes that $90 \%$ of line capacities is used by power flow transits related to the rest of Swiss and European transmission grid. The residual capacities dedicated for regional activities is decreased by a factor 10 (ie, $49 \mathrm{MW}$ ). Tthe deterministic version of ETEM-SG is solved with these reduced line capacities.

- In the third scenario, one take into account power flow transits using robust optimization 
techniques as described in Section 4 . One assumes a nominal saturation rate $\bar{\beta}_{l}=0.5$ and variability $\hat{\beta}_{l}=0.5$. This corresponds to a nominal capacity $\bar{c}_{l}=0.5 c_{l}$.

\subsection{Simulation results}

The results of the simulations, performed for a 2015-2050 horizon planning, are detailed below. The global evolutions of the energy system that is common to the three scenarios is first presented. Then the nodal power balance and prices are compared for the three scenarios in period 2050.

\subsubsection{Global energy system evolution}

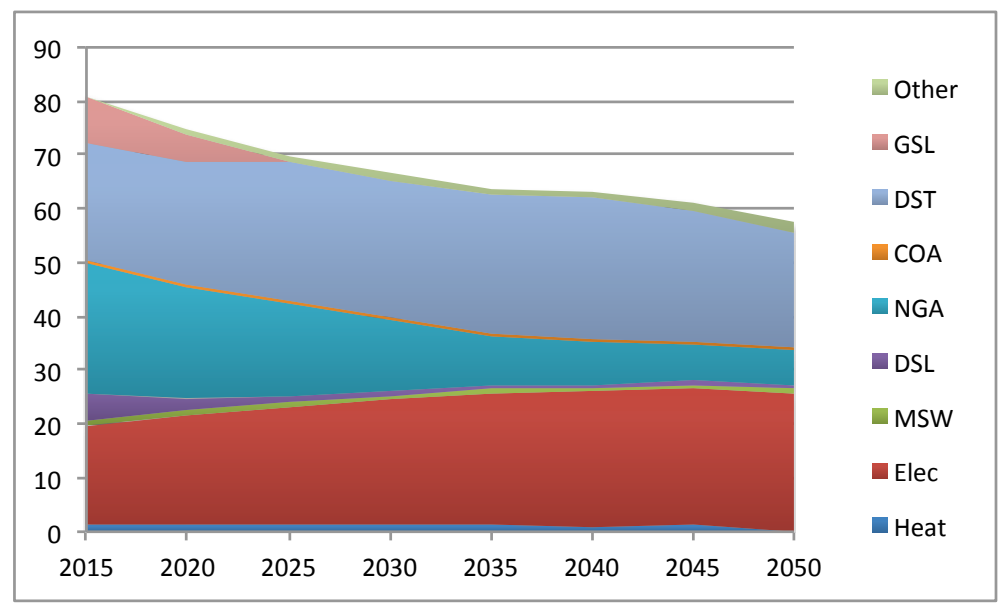

Figure 5: Energy mix (in PJ/year)

In the electricity sector, Figure 6 shows that wind mills technology is the main carbon free option to satisfy electricity consumption increase as well as environmental constraints. At global level, it can be observed that all scenarios lead to very similar situations. It seems that power flow constraints don't affect investments globally but have instead a significant impact at the nodal spatial scale, as shown in the next subsections. Figures 5 and 6 display the evolutions, observed for both scenarios, of regional energy mix and electricity production, respectively. On Figure 5, one notices an increase of electricity consumption, a reduction of gas use and a gasoline removal. Indeed, to meet the emissions constraint the model replaces

gas heaters by heat pumps in residential and building sectors and invest in hybrid and electric vehicles.

\subsubsection{Impact of transmission constraints on the 2050 electricity sector}

In this subsection one details the simulation results at each node and in particular on observes the impact of power transmission constrainta on nodal electricity balance and nodal pricing. 


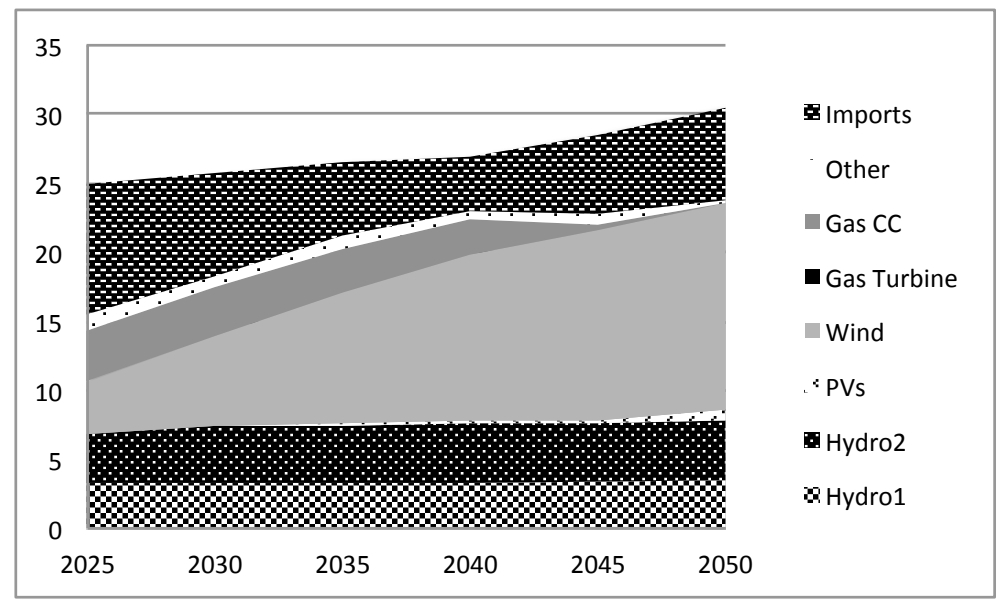

Figure 6: Electricity production mix (in PJ/year)

Nodal electricity balance in 2050. Figure 7 shows (in PetaJoules) for the three scenarios i) total electricity production and consumption at nodes, ii) annual electricity exchanges for local consumption on transmission lines and iii) total 2050 electricity imports at the three import nodes.

It can be observed that power flow constraints can have a significant impact on nodal electricity consumption and production patterns. For example, one observes for Verboix a change from 2.10 PJ in Scenario 1 to 6.12 PJ in Scenario 3 for electricity generation and from 5.86 to $10.35 \mathrm{PJ}$ for total electricity consumption. These different production and consumption patterns are explained by different technological choices at nodes. As expected power flow constraints affect strategic and operational decisions on the full energy system and not only on the electricity sector.

However, in practice the transmission grid is not fixed over time and new investments are usually performed to follow consumption and production evolution. Modelling of investment options on transmission lines would make it possible to increase line capacities and, as a consequence, would limit the changes in power consumption and production patterns.

Nodal electricity prices and congestion in 2050. Figure 8 summarises nodal electricity prices computed by ETEM-SG in 2050 in the three scenarios. It gives the minimum and maximum computed prices over the different timeslices. Figure 8 also displays the maximum utilisation rates of transmission lines over the different timeslices. In scenario 2, utilisation rates include interregional transits while in the robust scenario figures correspond to nominal utilisation rates without interregional transits.

First and as expected, in the first scenario with large transmission line capacities and without consideration of inter-regional power transit through the transmission grid, the network is oversized and nodal electricity prices are not affected by network congestion. In other words electricity prices are identical at all nodes and vary only in time. In the congested scenarios (scenarios 2 and 3), ETEM-SG generates very different nodal electricity prices. The highest prices are usually computed for central nodes that have no direct connection to imports. 
Surprisingly, the minimum electricity price in Verbois, that is connected to imports, is higher in the congested scenarios and higher than the import price. Indeed, this is the impact of power flow constraints. A marginal change in consumption in Verbois would modify the entire system and thus yields to an additional cost difficult to anticipate without a model like ETEM-SG.

Figure 8 demonstrates also a positive impact of robustness on nodal prices with maximum electricity prices in scenario 3 that are always lower than the ones computed in the two other scenarios. This results can be explained by the fact that robust optimization is a minmax approach and thus provides solutions that are robust for worst case situations, ie, congested network situations. Note that in this first tentative of applying robust optimization in that context, it was not assumed any known information on inter-regional power flow transit. With such information, one could deliver even more realistic and robust energy analysis.

\section{Conclusion}

In this paper, the multi-sectoral multi-energy long-term investment planning tool ETEM-SG has been extended to the consideration of power flow constraints in regional transmission grid by implementing a linearized DC power flow model that represents the transmission grid with the main constraints on the power flowing through the different arcs of the electricity transmission network. Then as power flows circulating in a regional transmission grid depend on what happens on the transmission grid for a much larger perimeter and it is uncertain, robust optimization technique is used to take into account the uncertainty on the capacity limits resulting from inter-regional transit.

The paper shows on a simple case study, that corresponds roughly to the "Léman Arc" region, the relevance of such a modelling exercise for long-term simulations of regional energy systems. First one can notice that power flow constraints together with line capacities have a significant impact on nodal electricity consumption and production patterns and thus on technological choices. Then one can observe the strong effect on maximum nodal prices when considering transit uncertainty using robust optimization.

Future works are now envisioned to allow the model ETEM-SG to provide realistic energy analysis to support decision makers. The first one would consist in coupling the regional ETEM-SG model with a national one (eg, Swissmod) in order to calibrate inter-regional power flow transit properly on simulation results performed with this much larger model. This cou-

pling exercise is not straightforward as it requires an harmonisation of both model in term of assumptions and technological evolutions. For example, regional and national models must provide compatible results in term of Electric Vehicle penetration as it has a significant impact on electricity grids. A second work will consist in extending the new transmission grid module in ETEM-SG to network investment decisions. This way ETEM-SG will then be able to make investment trade-off between grid capacity expansions and localized generation technologies. 


\section{References}

[1] C. Andrey, F. Babonneau, and A. Haurie. Modélisation stochastique et robuste de l'atténuation et de l'adaptation dans un système énergétique régional. application à la région midi-pyrénées. Nature Science Société, 23(2):133-149, 2015.

[2] F. Babonneau, M. Caramanis, and A. Haurie. Etem-sg: Optimizing regional smart energy system with power distribution constraints and options. ORDECSYS Technical report, 2016.

[3] F. Babonneau, A. Haurie, G. J. Tarel, and J. Thénié. Assessing the future of renewable and smart grid technologies in regional energy systems. Swiss Journal of Economics and Statistics, 148(2):229-273, 2012.

[4] F. Babonneau, A. Kanudia, M. Labriet, R. Loulou, and J.-P. Vial. Energy security : a robust programming approach and application to european energy supply via tiam. Environmental Modeling and Assessment, 17(1):19-37, 2012.

[5] F. Babonneau, O. Klopfenstein, A. Ouorou, and J.-P. Vial. Robust capacity expansion solutions for telecommunication networks with uncertain demands. Network, 62(4):255$272,2013$.

[6] F. Babonneau, J.-P. Vial, and R. Apparigliato. Robust optimization for environmental and energy planning. In J.A. Filar and A. Haurie eds., Uncertainty and Environmental Decision Making, Springer, 2010.

[7] R. Bacher. Power system models, objectives and constraints in optimal power flow calculations. In Bacher R. (Eds.) Frauendorfer K., Glavitsch H., editor, Optimization in Planning and Operation of Electric Power Systems, Lecture Notes of the SVOR/ASRO Tutorial Thun, Switzerland, October 14-16, 1992, pages 217-264, Heidelberg, May 1993. Physica-Verlag (Springer).

[8] A. Ben-Tal, L. El Ghaoui, and A. Nemirovski. Robust Optimization. Princeton University Press, 2009.

[9] A. Ben-Tal and A. Nemirovski. Robust convex optimization. Mathematics of Operations Research, 23:769-805, 1998.

[10] Berger C., Dubois R., Haurie A., Lessard E., Loulou R., and J.-P. Waaub. Canadian MARKAL: An advanced linear programming system for energy and environmental modelling. INFOR, 30(3):222-239, 1992.

[11] Fragnière E. and Haurie A. A stochastic programming model for energy/environment choices under uncertainty. Int. J. Environment and Pollution, 6(4-6):587-603, 1996.

[12] L. El-Ghaoui and H. Lebret. Robust solutions to least- square problems to uncertain data matrices. SIAM Journal of Matrix Analysis and Applications, 18:1035-1064, 1997.

[13] R. Loulou and M. Labriet. ETSAP-TIAM: the times integrated assessment model part i: Model structure. Computational Management Science, 5(1):7-40, 2008. 
[14] Office fédéral de l'énergie (OFEN). Die Energieperspektiven für die Schweiz bis 2050. 2012.

[15] ORDECSYS. Réseaux intelligents de transport/transmission de l'électricité en suisse. Technical report, ORDECSYS Technical report, 2013.

[16] ORDECSYS. Time of use (TOU) pricing: Adaptive and tou pricing schemes for smart technology integration. Technical report, ORDECSYS Technical report, 2014.

[17] P. A. Ruiz, J. M. Foster, A. Rudkevich, and M. C. Caramanis. Tractable transmission topology control using sensitivity analysis. IEEE Transactions on Power Systems, to appear.

[18] P. A. Ruiz, A. Rudkevich, M.C. Caramanis, E. Goldis, E. Ntakou, and R. Philbrick. Reduced mip formulation for transmission topology control. In USA University of Illinois at Urbana-Champaign, IL, editor, 50th Annual Allerton Conference on Communication, Control, and Computing, Monticello 2012.

[19] P.A. Ruiz, J.M. Foster, A. Rudkevich, and M. Caramanis. On fast transmission topology control heuristics. In Proc. 2011 IEEE Power and Energy Society General Meeting, Detroit, MI. IEEE, July 2011.

[20] A. L. Soyster. Convex programming with set-inclusive constraints and applications to inexact linear programming. Operations Research, 21:1154-1157, 1973.

[21] A.D.J. Stiel. Modelling liberalised power markets. Master's thesis, ETH Zürich, Centre for Energy Policy and Economics, September 2011.

[22] H. Weigt and I. Schlecht. Swissmod a model of the Swiss electricity market. Technical report, WWZ-Discussion Paper, 2014. 

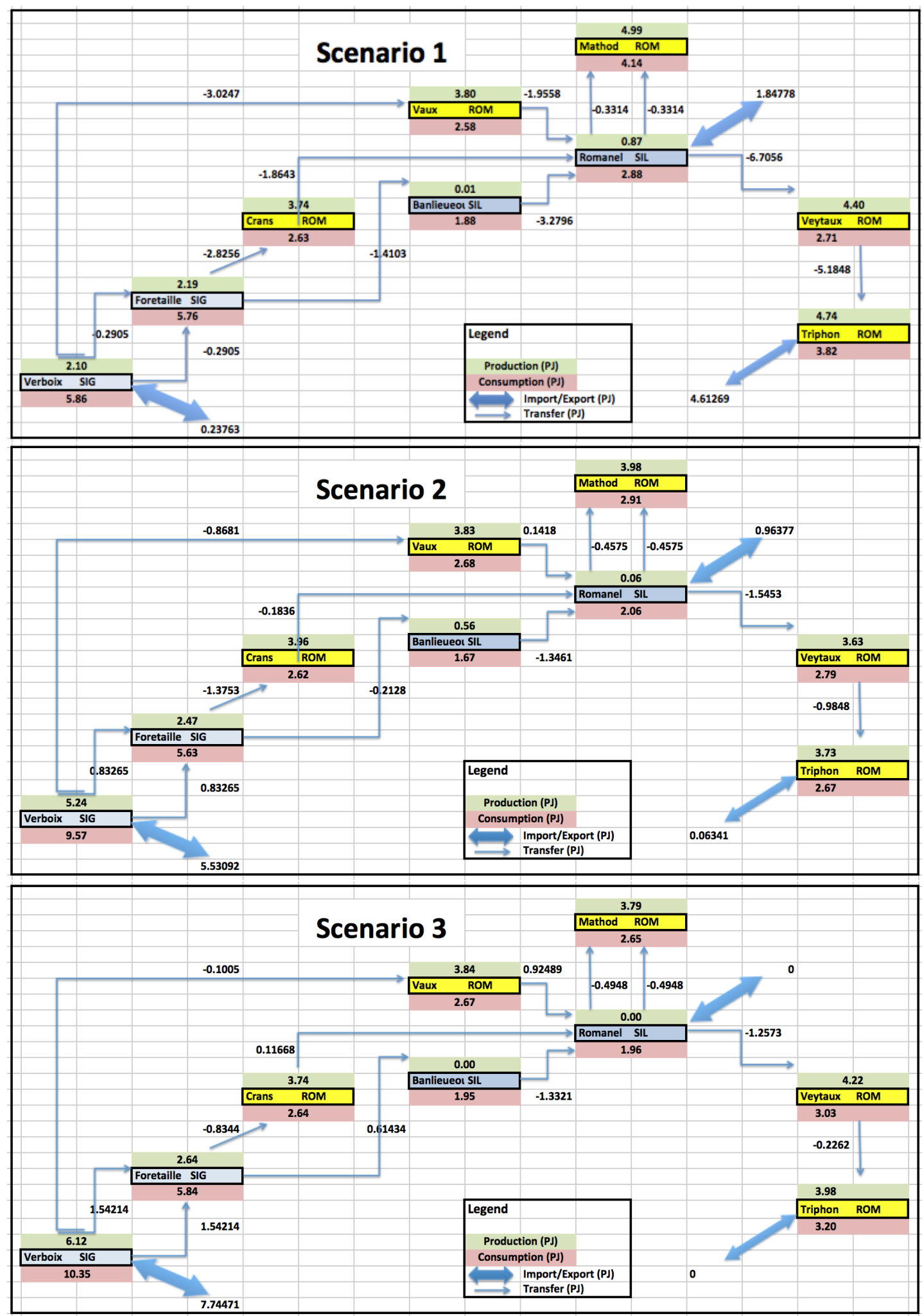

Figure 7: Nodal electricity balance in 2050. 

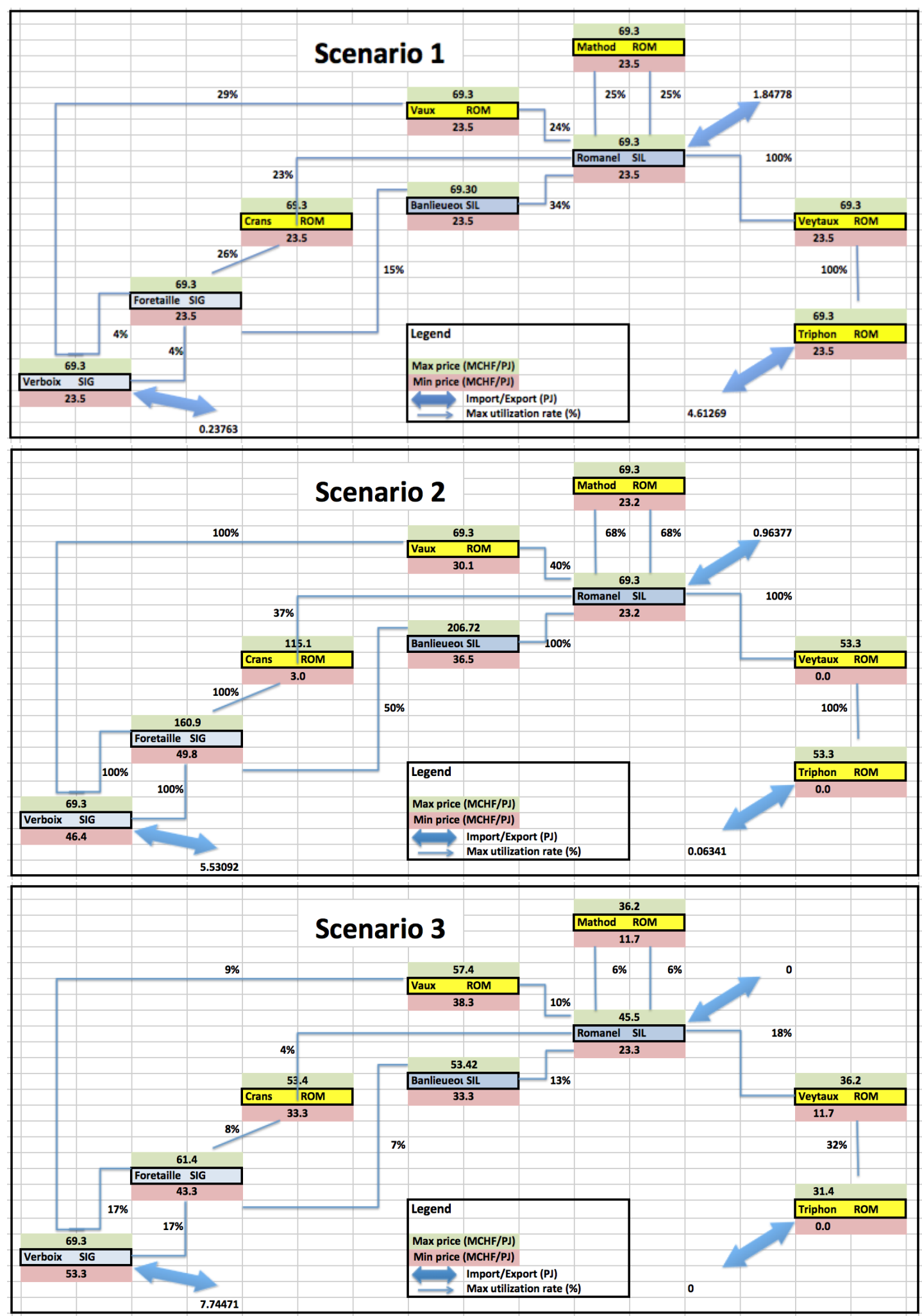

Figure 8: Statistics on nodal electricity prices (in MCHF/PJ) and on line utilization rate (in \%) in 2050 . 